\begin{tabular}{lcccr} 
T H E A R C H I V E & O F & M E C H A N I C A L & E N G I N E E R I N G \\
\hline VOL. LX & 2013 & Number 2 \\
10.2478/meceng-2013-0018 &
\end{tabular}

\author{
MIROSEAW KABACINSSKI *, CYPRIAN T. LACHOWICZ ${ }^{* *}$, JANUSZ POSPOLITA ***
}

\title{
NUMERICAL ANALYSIS OF FLOW AVERAGING TUBES IN THE VORTEX-SHEDDING REGIME
}

\begin{abstract}
This paper presents a study on flowmeters oscillatory motion when subjected to periodical, enforced vibrations induced by vortex-shedding. The proposed mathematical modelling of flow was compared to corresponding numerical simulation, in form of modal analysis. The frequencies of vortices generation and detachment were calculated for a number of flow velocities in two different flowmeter profile variants. The performed modal evaluation enabled estimating their natural frequencies, and in consequence the acquired data helped us to determine flow velocity for which the analyzed structures were prone to resonant vibrations.
\end{abstract}

\section{Introduction}

Flowmeters with flow averaging tubes (Fig. 1) are becoming more and more common in industrial practice. They are particularly applicable for measurements of flows in large diameter pipelines. This comes as a consequence of their competitiveness in terms of unit cost and simpler installation in comparison to other possible solutions. In addition, flowmeters with flow averaging tubes do not introduce significant additional permanent pressure losses. For the case of flowing media with high temperatures, they offer virtually the only possibility of measuring fluid flow [1].

* Department of Thermal Engineering and Industrial Facilities, Opole University of Technology,ul. Mikołajczyka 5, 45-271 Opole, Poland; E-mail: m.kabacinski@po.opole.pl

** Department of Mechanics and Machine Design, Opole University of Technology, ul. Mikołajczyka 5, 45-271 Opole, Poland

*** Department of Thermal Engineering and Industrial Facilities, Opole University of Technology, ul. Mikołajczyka 5, 45-271 Opole, Poland 


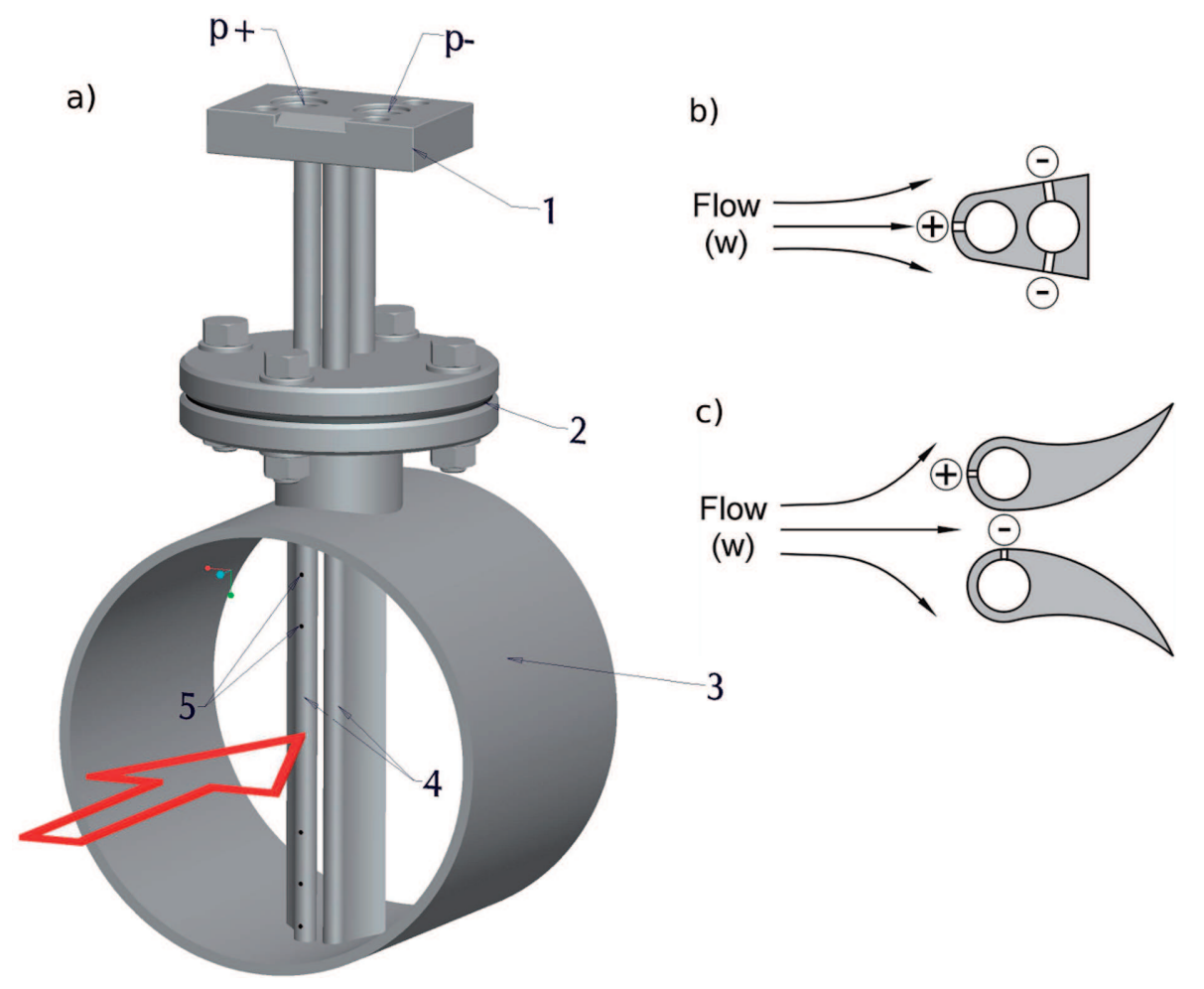

Fig. 1. Flowmeter with Flow Averaging Tube (FAT): a) general view (1 - head, 2 - flange fittings, 3 - pipeline, 4 - two-profile flowmeter, 5 - impulse holes), b) cross-sections of streamlined flowmeter, c) cross-section of two-profile flowmeter

In the discussed flowmeters, the relation between the mean flow velocity and measured differential pressure in impulse chambers $A_{i} p$ takes the form

$$
w=K \sqrt{\frac{2 \Delta p}{\rho}} .
$$

In the above formula, $K$ is the flow coefficient through a tube and $\Delta p=p^{+}-p^{-}$. The pressures $p^{+}$and $p^{-}$are averaged pressures in the impulse chambers. One of the problems that is encountered during exploitation is the one associated with vibrations of probes. They occur as a result of dynamic effect of the stream on probe surface. As a result, measurement range of a probe is worsened and the measurement uncertainty grows. This issue is particularly detrimental when frequencies generated by the vortex probe correspond to the self-induced vibrations of the probe. For these reasons, probe manufacturers determine the ranges of measured streams and frequencies of fitting probes for specific pipeline diameters. This is done with the purpose of controlling the conditions for which frequencies of the dynamic effect approach self- 
induced vibrations of the probes. The frequencies of vibrations are determined on the basis of conducted measurements. For instance, extensometers can be used to determine a periodically variable force affecting a probe. Literature on this subject can also provide values of Strouhal number for selected profiles. In this case, a similarity number relates a specific dimension of a given profile with the mean velocity and frequency of generated vortices. Exemplary information regarding two such profiles can be found in [2]. However, the phenomenon of stream passage through a profile has physically complex characteristics and the effect of a fluid on a profile is a three-dimensional and physically complex phenomenon due to generated vortices. Hence, an attempt in this paper is concerned with mathematical modelling and numerical simulation of this issue. The analysis here is concerned with two types of probes - one- and two-profile ones. Their crosssections and dimensions are presented in Fig. 7. The two-profile probe gives a considerably higher measured pressure interval in comparison to other flowmeters [3], [4], [5], [6]. As a result, it is possible to reduce uncertainty of measurements, in particular with regard to gas streams where differential pressure increases.

\section{Mathematical model of fluid flow in the vicinity of a probe}

The analysis involved isothermal $\left(t=20^{\circ} \mathrm{C}\right)$, turbulent, non-stationary two dimensional $(2 D)$ flow of viscous fluid that does not display compressible characteristics (water, air). For the velocities of air flow not exceeding $30 \mathrm{~m} / \mathrm{s}$, the assumption of non-compressibility is justified. The mathematical model is described for the components $\bar{U}_{i}$ of the velocity vector [7]

$$
\rho \frac{\partial \bar{U}_{i}}{\partial t}+\frac{\partial\left(\rho \bar{U}_{i} \bar{U}_{j}\right)}{\partial x_{j}}=-\frac{\partial p}{\partial x_{i}}+\frac{\partial}{\partial x_{j}}\left[\mu_{e f}\left(\frac{\partial \bar{U}_{i}}{\partial x_{j}}+\frac{\partial \bar{U}_{j}}{\partial x_{i}}\right)\right]
$$

and based on continuity equation

$$
\frac{\partial \rho}{\partial t}+\frac{\partial\left(\rho \bar{U}_{i}\right)}{\partial x_{i}}=0
$$

In the above equations $\bar{U}_{i}$ is a component of velocity vector in the respective $x$ and $y$ directions, $\rho$ - fluid density. The quantity $\mu_{e f}$ represents effective viscosity that is given by the total of molecular viscosity $\mu$ and turbulent one $\mu_{t}$, that is

$$
\mu_{e f}=\mu+\mu_{t}
$$


Turbulent viscosity is determined on the basis of $R N G^{*} k-\varepsilon$ [8] turbulence model

$$
\mu_{t}=\rho C_{\mu} \frac{k^{2}}{\varepsilon}
$$

in which $C_{\mu}$ is equal to 0.0845 for the case of $R N G k-\varepsilon$ model for developed turbulence. The kinetic energy of turbulence $k$ and its dissipation rate $\varepsilon$ are determined on the basis of transport equations, which for the case of RNG $k-\varepsilon$ model take the form [7]:

$$
\begin{gathered}
\frac{\partial k}{\partial t}+\bar{U}_{i} \frac{\partial k}{\partial x_{j}}=\frac{1}{\rho} \frac{\partial}{\partial x_{j}}\left(\alpha_{k} \mu_{e f} \frac{\partial k}{\partial x_{j}}\right)+\frac{\mu_{t}}{\rho}\left(\frac{\partial \bar{U}_{i}}{\partial x_{j}}+\frac{\partial \bar{U}_{j}}{\partial x_{i}}\right) \frac{\partial \bar{U}_{i}}{\partial x_{j}}-\varepsilon \\
\frac{\partial \varepsilon}{\partial t}+\bar{U}_{i} \frac{\partial \varepsilon}{\partial x_{j}}=\frac{1}{\rho} \frac{\partial}{\partial x_{j}}\left(\alpha_{\varepsilon} \mu_{e f} \frac{\partial \varepsilon}{\partial x_{j}}\right)+\frac{C_{1} \mu_{t} \varepsilon}{\rho k}\left(\frac{\partial \bar{U}_{i}}{\partial x_{j}}+\frac{\partial \bar{U}_{j}}{\partial x_{i}}\right) \frac{\partial \bar{U}_{i}}{\partial x_{j}} \\
-\left(C_{2}+\frac{C_{\mu} \eta^{3}\left(1-\eta / \eta_{\circ}\right)}{1+\beta \eta^{3}}\right) \frac{\varepsilon^{2}}{k}
\end{gathered}
$$

where

$$
\eta=\frac{s k}{\varepsilon}, s=\sqrt{2 s_{i j} s_{i j}}, \quad s_{i j}=\frac{1}{2}\left(\frac{\partial \bar{U}_{i}}{\partial x_{j}}+\frac{\partial \bar{U}_{j}}{\partial x_{i}}\right) .
$$

In Eqn. ((6)) and ((7)) $\alpha_{k}$ and $\alpha_{\varepsilon}$ denote the reversed Prandtl numbers for $k$ and $\varepsilon$, respectively, which for higher values of $\operatorname{Re}\left(\mu / \mu_{e f} \ll 1\right) ; \eta_{0}=4.38$, $\beta=0.012 C_{1}=1.42, C_{2}=1.68, \alpha_{k}=\alpha_{\varepsilon}=1.3929$.

Equations $((1))$ to $((6))$ form a complete system, which defines the examined flow phenomenon. Respective boundary and initial conditions are combined with this system of equations for all variables. The computational domain, as well as conditions on its boundaries, are presented in Fig. 2.

The computational domain adopts such distributions of all variables, which are presented in its inlet cross-section. The boundary conditions for $k$ and $\varepsilon$ on the walls depend on the applied $k-\varepsilon$ model. The equations of the mathematical model are solved with the Finite Volume Method using ANSYS/FLUENT software [7]. The discretization of the calculation region was undertaken with ANSYS/GAMBIT software [9].

\section{Results of calculations}

Figure 3 presents numerically-determined velocity distributions at selected instant for the two examined probes. A clear vortex path is generated by

* Re-Normalisation Group 


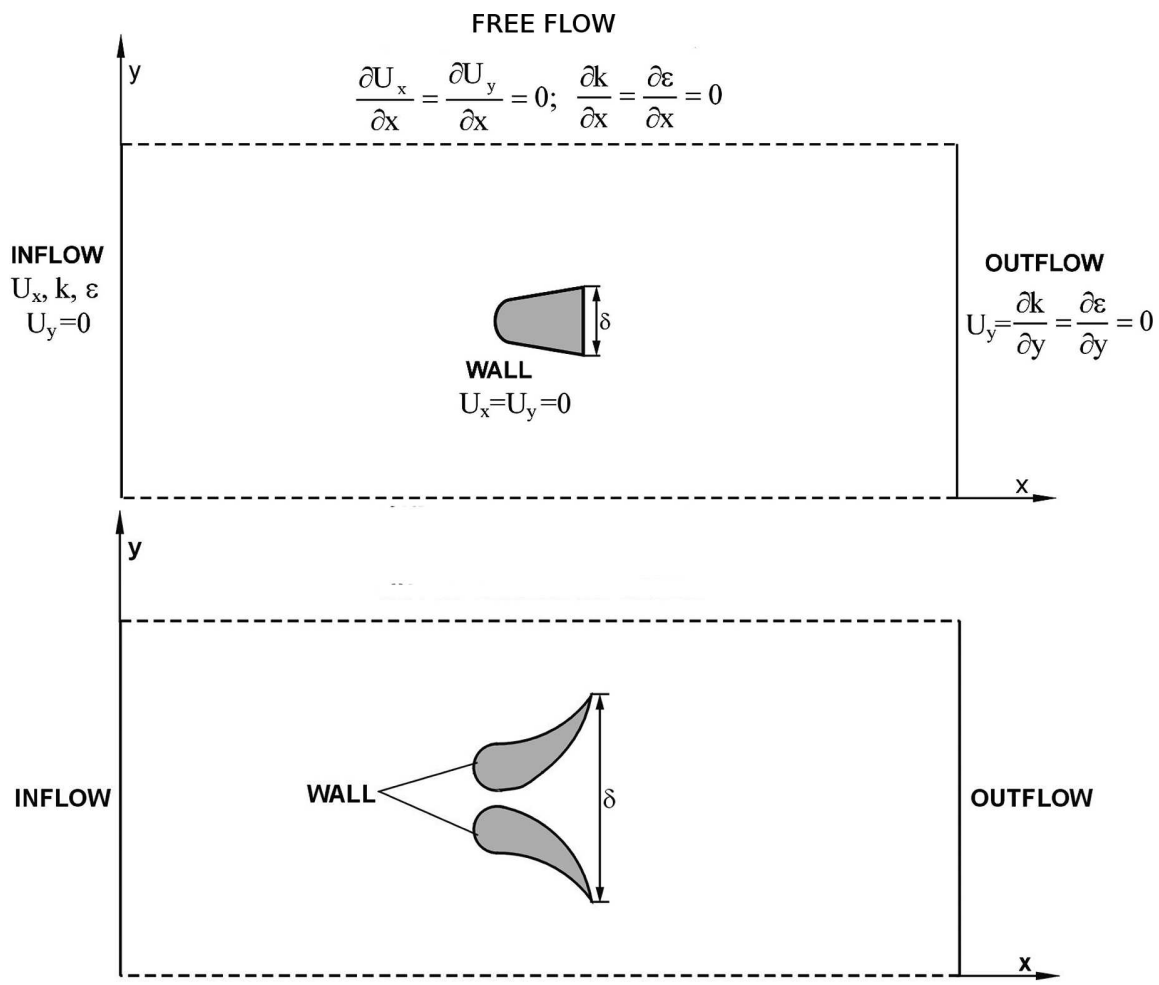

Fig. 2. Boundary conditions for the examined flow system layout: a) one-profile probe,

b) two-profile probe

the profile. This path is generated beyond the differential pressure measurement points, which leads to a virtually unchanged measured value of the differential pressure $\Delta p$. Largest pressure differences are noted beyond the rear side of the profile.

The generated vortex path leads to periodical effect of the fluid on the flowmeter probe. If the frequency of this effect corresponds to the frequency of self-induced vibrations of the installed probe, there is a hazard of both deterioration of its metrological parameters as well as its complete failure. The frequency of the generated vortices rises with the increasing flow velocity. This relation is expressed with the Strouhal similarity number $S t=f \delta / U$ ( $f$ - frequency of generated vortices, $\delta$ - characteristic dimension and $U-$ velocity of the fluid flow) and Reynolds number $R e=U \delta / v$. For the case of one-profile probe, the characteristic dimension $\delta$ is the width of the rear wall (outflow side) of the probe ( $\delta=13.6 \mathrm{~mm}$ ), while for a two-profile probe the width of the projection of the profile in the direction of the flow was adopted to be the value of $\delta=43.5 \mathrm{~mm}$. Velocity $U$ is the velocity of the stream inflow with a uniform velocity on the examined profile. Fig. 4 presents numerically determined relation between these two quantities for both examined probes, 


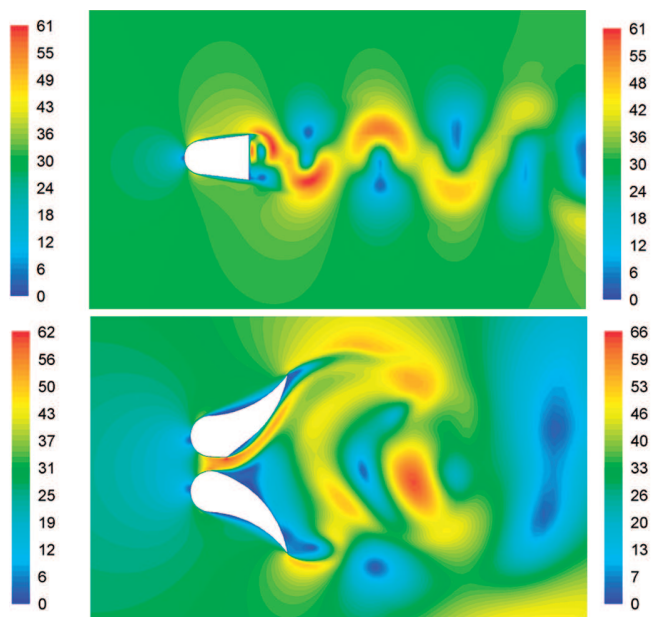

a)
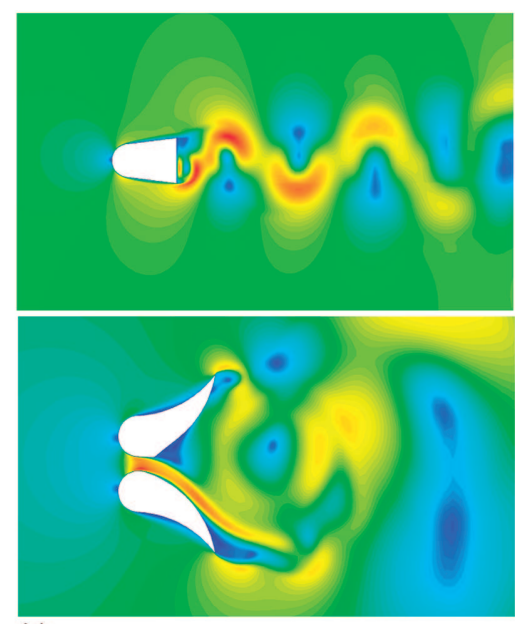

b)

Fig. 3. Velocity distributions $(\mathrm{m} / \mathrm{s})$ around the selected probes for two instants $t_{1}$ and $t_{2}$ (b) for mean velocity at the inlet $\mathrm{w}=30 \mathrm{~m} / \mathrm{s}$

illustrating that in each of the cases the Strouhal number varies inconsiderably. It proves the proportionality of vortex separation relative to the velocity of medium affecting a probe.

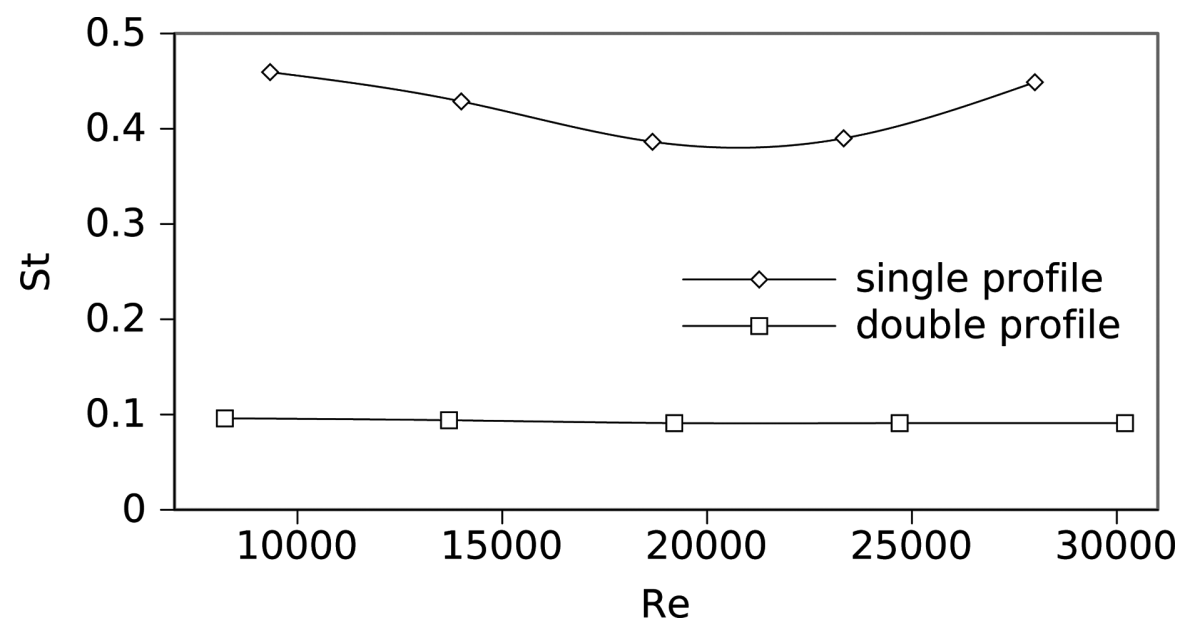

Fig. 4. Strouhal number $S t$ for the examined probes in the function of Reynolds number $R e$

For the purposes of further examinations mean values of Strouhal number were adopted for the examined profiles: $S t=0.093$ for two-profile and $S t=0.423$ for one-profile probes, respectively. 


\section{Finding the flowmeter's eigenvalues}

Dynamical loadings, caused by the detachment of vortices, are followed by periodically changing displacements of the flowmeter [10], [11]. These, under specific flow conditions, take form of enforced vibrations [12], [13]. In this paper, the fluid-structure interaction is not taken into account. A decision was made to separately analyze flow around a sensor cross-section and independently determine the frequency of vibrations involved in it. Solid state mechanics states that such motion in linear systems should be modelled with simplified concentrated mass distribution. An example that simply illustrates that, is a single degree of freedom structure (Fig. 5) consisting of mass $m$ constrained to the base by means of a spring, of $k_{s t}$ stiffness.

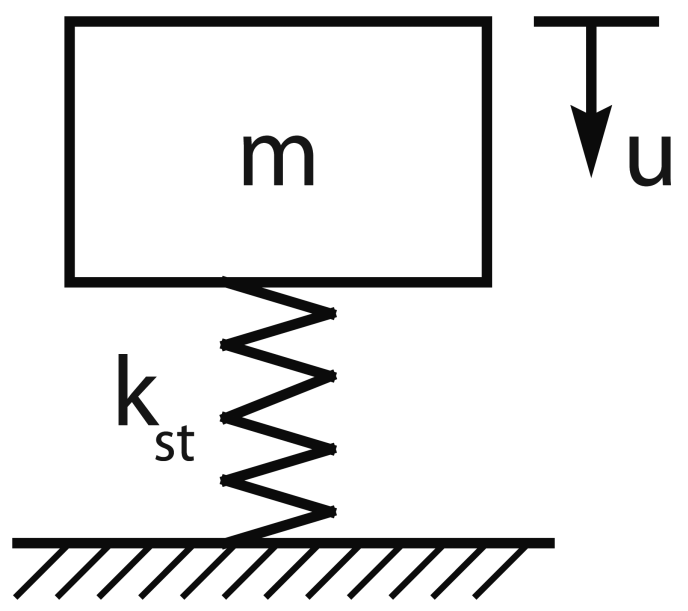

Fig. 5. A model of oscillating, single degree of freedom structure

The description of the undamped natural vibrations of such system can be presented by following differential equation:

$$
m \ddot{u}(t)+k_{s t} u(t)=0,
$$

in which $u(t)$ and $\ddot{u}(t)$ are respectively, the displacement and acceleration of considered mass. The adopted computational model of flowmeter is essentially a structure of $n$ concentrated masses with springs, connected in series, as presented on Fig. 6.

When utilizing the finite element method [14] to analyze the flowmeter's behaviour, it is necessary to reformulate the differential equations of motion into their matrix form [15], [16]

$$
[M]\{\ddot{u}(t)\}+\left[K_{s t}\right]\{u(t)\}=0,
$$




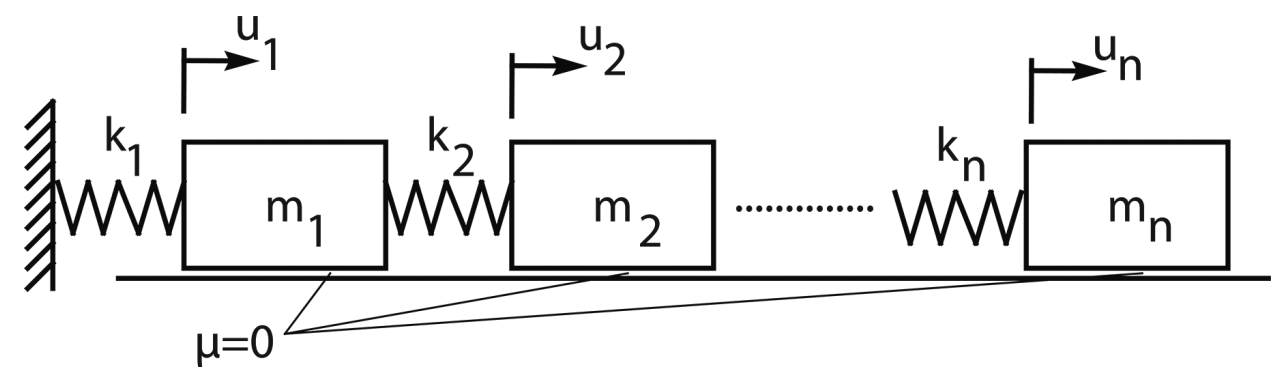

Fig. 6. Idealization of flowmeter as $n$-degree of freedom, undamped linear system

in which, $[M]$ is the mass and $\left[K_{s t}\right]$ the stiffness matrices of the model, while $\{\ddot{u}(t)\}$ and $\{\mathrm{u}(\mathrm{t})\}$ represent, respectively, the acceleration and displacement vectors of the flowmeter model. Solving Eqn. (10) with FEM methodology causes the mass and stiffness matrices to be generated automatically, on a basis of geometrical model and material properties. Since the modal analysis was performed for a linear solid body model, only two material constants Young's modulus E and Poisson ratio are required for calculations. Way of finding Eqn. 9 depends on adopting a particular form of solution

$$
\{u(t)\}=\{\phi\} \sin \omega t
$$

where: $\{\phi\}$ is the eigenvector and the radian frequency $(\omega=2 \pi f)$. Assuming that the solution to Eqn. (11) is harmonic, one may observe that the motion of all unconstrained nodes in the structure is synchronized. This implies that the structure resonant mode shapes are constant in time, and dependent only on the model's geometry, mass distribution and boundary conditions id est, the fixing method. By differentiating expression (Eqn. (11)) and substituting the results into Eqn. (10), the following formula is obtained

$$
-\omega^{2}[M]\{\phi\} \sin \omega t+\left[K_{s t}\right]\{\phi\} \sin \omega t=0,
$$

and after some basic transformations,

$$
\left(\left[K_{s t}\right]-\omega^{2}[M]\right)\{\phi\}=0 .
$$

the resulting expression, called the eigenvalue equation, is a set of algebraic equations, which can be reduced by means of linear algebra to following form [13]

$$
(G-\lambda I) x=0 .
$$

In the above equation [G] is a square matrix, $\lambda$ is the eigenvalue $\omega^{2}=\lambda, I$ represents the unit matrix, while $x$ stands for eigenvector $(\{\phi\}=x)$. Two distinct 
solutions are possible for equation $((14))$ and ipso facto for formula (Eqn. (13)) as well. The first solution states that $\{\phi\}=0$, therefore it is trivial and has no practical application, whereas the second solution implies that if determinant of the matrix $\left[K_{s t}\right]-\omega^{2}[M]$ is zero, then a significant solution $\{\phi\} \neq 0$ exist. For a discrete set of eigenvalues $\omega_{i}^{2}$ with corresponding eigenvectors $\left\{\phi_{i}\right\}$, the determinant equals zero. Each eigenvalue with matching eigenvector are associated with a modal frequency $\left(f_{i}=\omega_{i} / 2 \pi\right)$ which, in turn, corresponds to a distinct resonant mode shape. It is important to acknowledge that the calculated values that describe the geometrical form of modal shapes, are relative and scaled to the highest value found, thus can only be used for comparative purposes. It is a fallacy to regard such results as the observed flowmeter's displacements, since those should be rather associated with a possible shape of deformations in the structure during a resonance. The adopted interpretation of displacement, which corresponds to a particular modal shape, is a consequence of a fact that natural vibrations are caused by deflection from the equilibrium position, when the body is not subjected to any form of external excitation, other than that which sustain the balance or seek to restore it. The amplitudes of flowmeter's oscillatory motion are dependent of the initial value of deflection (potential energy) or the initial velocity (kinetic energy) given to the structure. Consequently, first modal shape corresponds to the lowest value of either the potential or kinetic energy, while exciting the body to subsequent mode shapes require higher energy input.

\section{The numerical result}

First step in preparation of modal analysis is constructing geometrical models of both flowmeters types. FEMAP software aided the modelling process and exemplary models $(100 \mathrm{~mm}$ in length), together with generated finite element mesh are presented in Fig. 7.

The before-mentioned mesh structure consisted of hexaedral elements, each incorporating 20 nodes. For both types of flowmeters, two distinct fixing methods were developed. First approach was based on fixing the model only on one side, leaving the other unconstrained (Fig. 8a). The second method limited both ends of the flowmeter, but while one side was fully fixed, displacements on the other side were restrained from any other direction but along $\mathrm{X}$ axis (Fig. 8b).

In the case of the double profile flowmeter, mounting was ensured by including a special mould component model. In real-life structure, profiles are pressed into this part, which serves as mounting base for the entire device. 

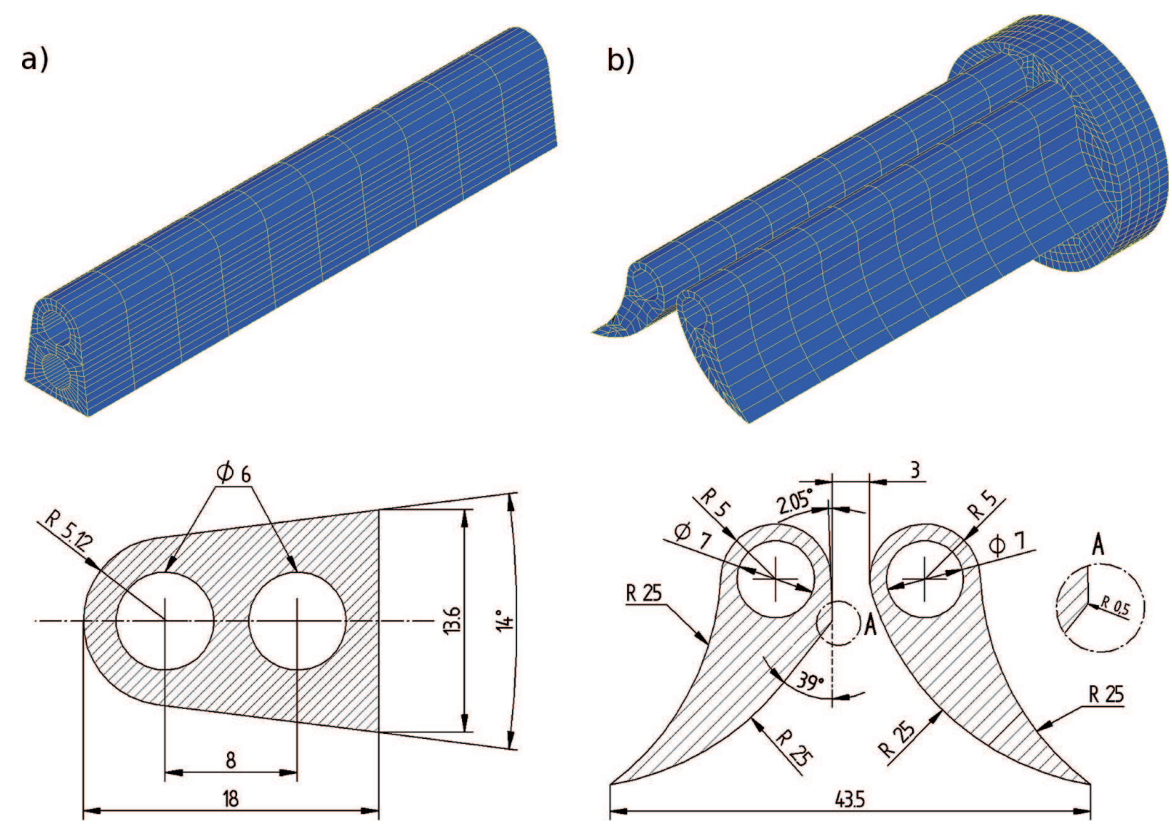

Fig. 7. Numerical models (geometry with finite element mesh structure) and cross sections of: a) single profile flowmeter, b) double profile flowmeter

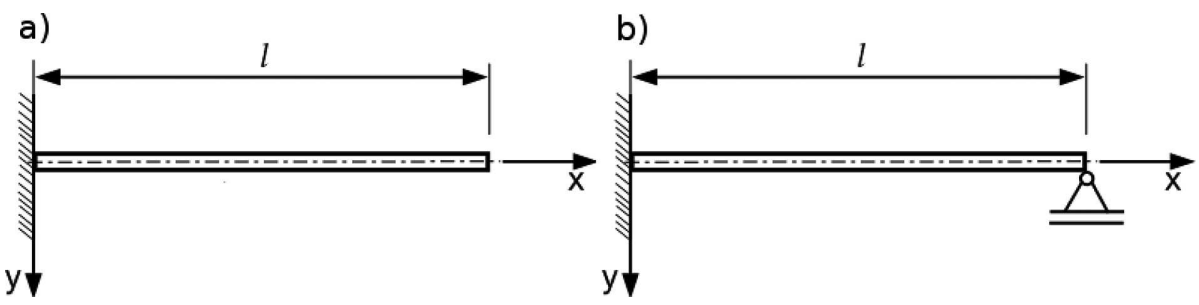

Fig. 8. Methodology for fixing the flowmeters: a) single side (unilaterally) b) both sides (bilaterally)

In corresponding numerical model fixing points were set along the lateral surface of the mould cylinder, as presented on Fig. 9.

Material properties are a key factor in finding system eigenvalues and associated mode shapes. $0 H 18 N 9$ steel grade basic properties are - the Young's modulus of $193 \mathrm{GPa}$, Poisson's ratio of 0.27 and density of $8000 \mathrm{~kg} / \mathrm{m}^{3}$. Material tensile strength $R_{m(\min )}$ is $485 \mathrm{MPa}$ whereas the yield strength $R_{e(\min )}$ stands around $170 \mathrm{MPa}$. Figures 10 and 11 show exemplary mode shapes for $300 \mathrm{~mm}$ long, single and double profile flowmeter variants. Numerical models presented in mentioned figures were unilaterally fixed. The values presented in displacement bars seen on Figures 10 and 11 are to be interpreted as specified in closing paragraph of chapter 4 . Since the mounting plate is an 


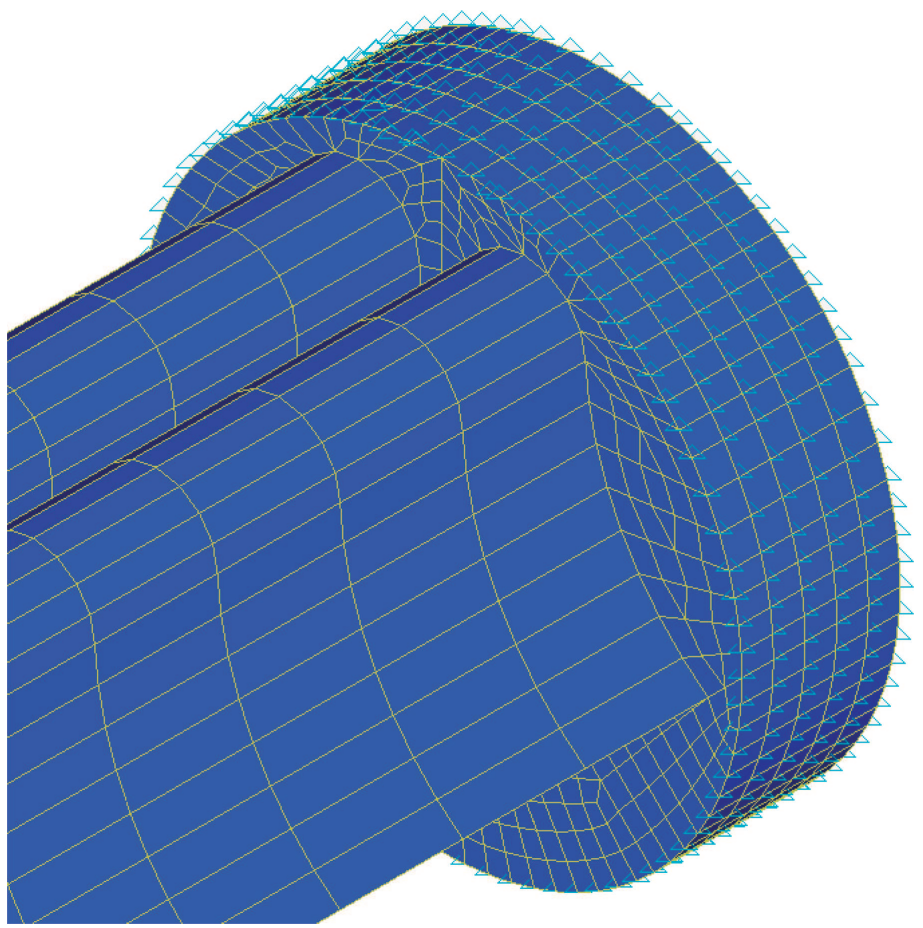

Fig. 9. Double-profile flowmeter fixe to mounting plate

integral part of the device, the calculated resonant frequencies and mode shapes apply to the flowmeter as a whole. Additional numerical evaluation found that modal characteristic of each profile, when tested independently, differ only slightly, so in further considerations system's common resonant frequency was taken into account.
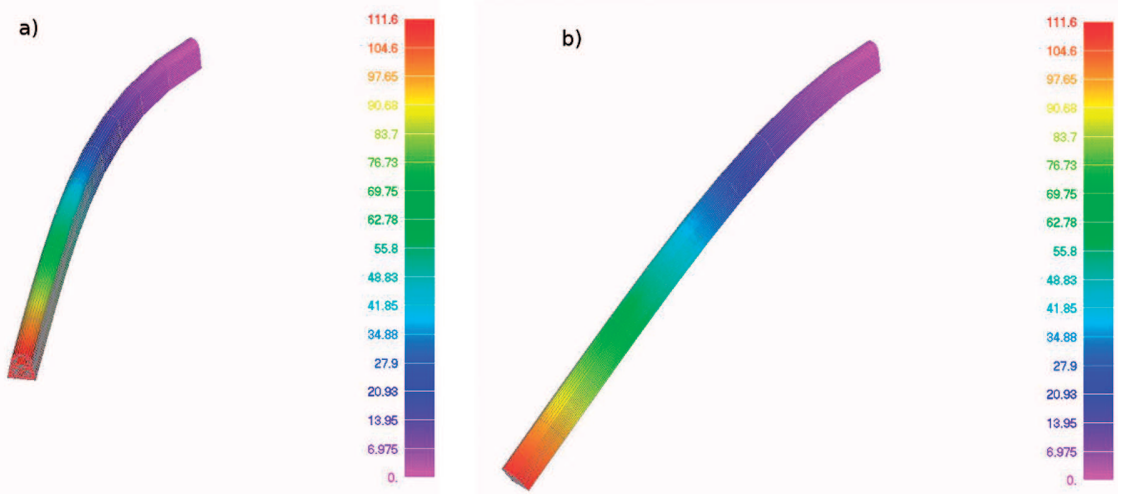

Fig. 10. Mode shape of a single profile flowmeter, for a) first modal frequency b) second modal frequency when mounted from single side 

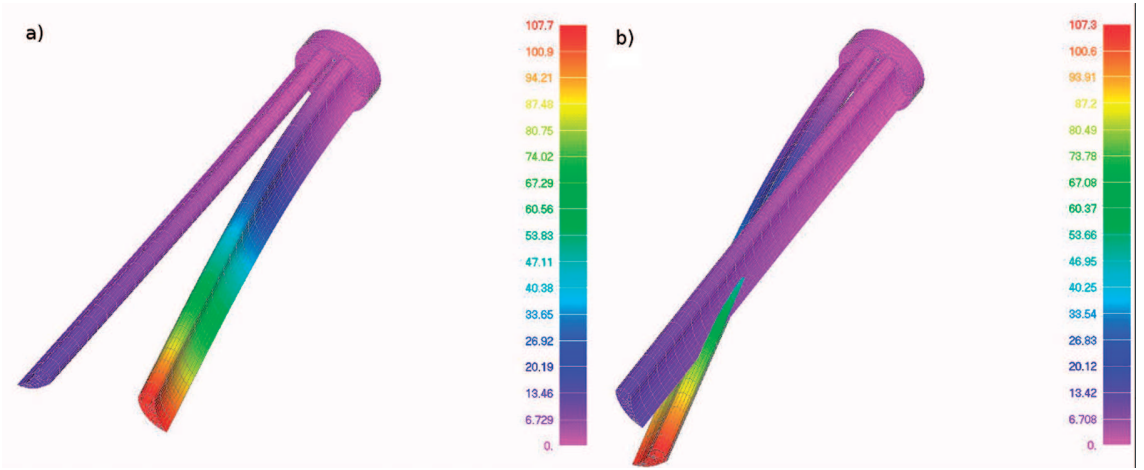

Fig. 11. Mode shapes of a double profile flowmeter, for a) first modal frequency b) second modal frequency when mounted from single side

Table 1 presents lowest modal frequencies (first mode) for both flowmeter types. Further resonant frequencies, corresponding to second and fourth mode are over a dozen times higher than the base frequency. On the basis of mean value of Strouhal number $S t$, the fluid flow speed was calculated, for which the frequency of vortices creation and shedding match the first modal frequency of given flowmeter length and type.

Table 1.

Base resonant frequencies (first modes) and corresponding stream velocities that induce the resonance effect

\begin{tabular}{|c|c|c|c|c|c|c|c|c|}
\hline Flowmeter & \multicolumn{4}{|c|}{ unilateral fixation } & \multicolumn{3}{c|}{ bilateral fixation } \\
\hline length & single profile & \multicolumn{2}{|c|}{ double profile } & \multicolumn{2}{c|}{ single profile } & \multicolumn{2}{c|}{ double profile } \\
\hline & $f$ & $U$ & $f$ & $U$ & $f$ & $U$ & $f$ & $U$ \\
\hline$m m$ & $H z$ & $m / s$ & $H z$ & $m / s$ & $H z$ & $m / s$ & $H z$ & $m / s$ \\
\hline & 1061.0 & 360.7 & 662.9 & 148.3 & 4325.0 & 1434.6 & 2857.0 & 639.1 \\
\hline & 268.0 & 91.1 & 170.2 & 38.1 & 1152.0 & 382.1 & 742.5 & 166.1 \\
\hline & 119.2 & 40.5 & 76.0 & 16.9 & 518.3 & 171.9 & 334.5 & 74.8 \\
\hline & 67.1 & 22.8 & 43.1 & 9.6 & 292.7 & 97.1 & 188.0 & 42.0 \\
\hline & 42.9 & 14.5 & 27.5 & 6.1 & 187.0 & 62. & 120.0 & 26.84 \\
\hline & 29.8 & 10.1 & 19.2 & 4.3 & 130.5 & 43.3 & 84.0 & 18.8 \\
\hline & 21.9 & 7.4 & 14.1 & 3.2 & 95.9 & 31.8 & 62.0 & 13.8 \\
\hline & 16.7 & 5.7 & 8.6 & 1.9 & 73.5 & 24.4 & 47.5 & 10.6 \\
\hline & 13.2 & 4.5 & 5.7 & 1.3 & 58.0 & 19.2 & 37.5 & 8.3 \\
\hline & 10.7 & 3.6 & 4.8 & 1.1 & 47.0 & 15.6 & 30.4 & 6.8 \\
\hline
\end{tabular}


Figure 12 illustrates the relation between the length of flowmeter and calculated flow velocity, while table 1 lists precise values of $U$ with corresponding excitation frequencies.

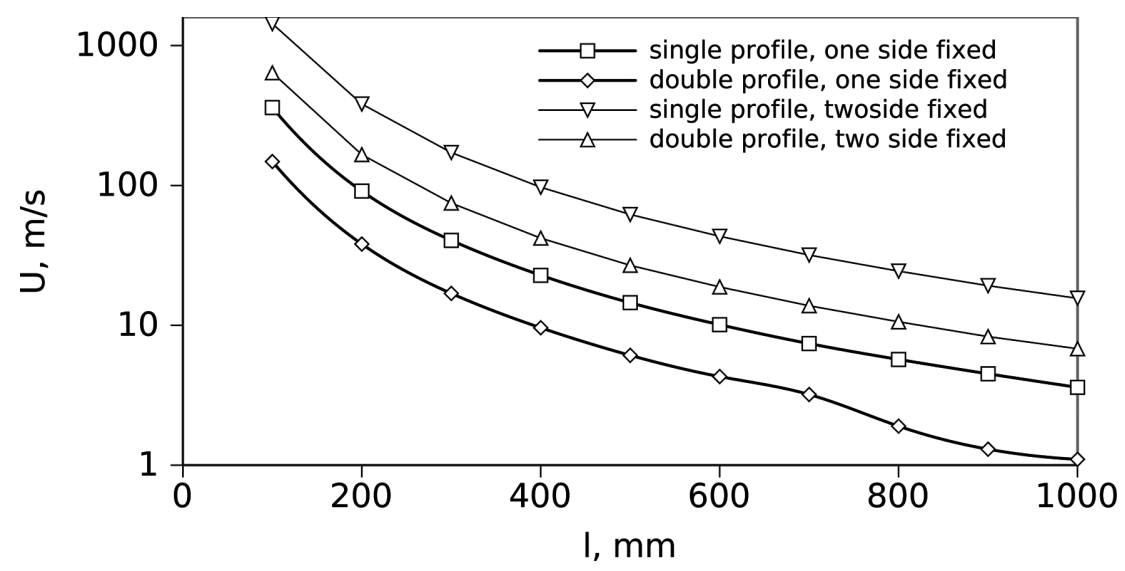

Fig. 12. Mean values of stream velocity that induces vortices, against flowmeter length

The above characteristic shows that the stream flow rate decreases as the length of the flowmeter increases, so device vibrations are in fact an operational issue, especially in longer flowmeters, where the frequency of vortices detachment overlaps its natural frequencies. These phenomena may cause enforced vibrations in structure, possibly oscillatory motion of frequency near the resonance spectrum. Counter-measuring that effect can be achieved by changing the fixing method, so that both ends of the flowmeter are supported (as described previously). Another method would require altering the geometrical profile to a similar shape, but with an increased moment of inertia. Figure 12 clearly shows that single-sided constraining, and high flow speeds are feasible only for shorter flowmeters, ranging $100-200 \mathrm{~mm}$ in length, longer devices require both ends to be supported. When installing these flowmeters in pipes of larger diameter, it is necessary to utilize second supporting method (as described previously) and increasing the flowmeter's cross-sectional area.

\section{Conclusions}

In the course of this paper, a mathematical model and numerical calculations of viscous fluid flow around a flowmeter were presented. The main focus of this work were the effects occurring during generation and detachment of vortices around the device, which are caused by periodically changing pressure distribution, potentially exciting the flowmeter to resonant vibrations. This is of crucial significance, when the frequency in which 
the vortices detach from flowmeter's surface overlaps its modal frequencies. A method for calculating the dangerous modal frequencies with corresponding flow velocities was introduced and presented with some exemplary data (for flowmeters that average the dynamical pressure of fluid [17]). Working ranges that consider the modal limitations, were found for all analyzed types of flowmeters. The issues concerning the detachment of vortices in relation to flow speeds and modal characteristic of flowmeter are of great importance, especially in regard to high-diameter piping systems.

Manuscript received by Editorial Board, March 27, 2012;

final version, April 18, 2013.

\section{REFERENCES}

[1] Spitzer D.: Flow Measurement: practical guides for measurement and control. Research Triangle Park, 1991.

[2] Baker R. C.: Flow Measurement Handbook. University of Cambridge, 2000.

[3] Węcel D., Chmielniak T., Kotowicz J.: A matematical model of the self-averaging Pitot tube. A mathematical model of a flowmeter, Flow Measurement and Instrumentation (19), 2005, pp. 301-306.

[4] Preso, Technical documentation. http:// www.preso.com

[5] Kabaciński M., Pospolita J.: Numerical and experimental research on new cross-sections of averaging pitot tubes, Flow Measurement and Instrumentation (19), 2008, pp. 17-27.

[6] Kabaciński M., Lachowicz C. T., Pospolita J.: Analysis of flowing fluid effect on flow averaging. TASK Quarterly, Vol 12(3), 2008, pp. 217-226.

[7] ANSYS/Fluent, Fluid Dynamics Analysis Package. Fluid Dynamics International Inc., 2010.

[8] Dobrowolski B., Kabaciński M., Pospolita J.: A mathematical model of the self-averaging pitot tube. a mathematical model of a flow flowmeter, Flow Measurement and Instrumentation (16), 2005, pp. 251-256.

[9] ANSYS/Gambit, Fluid Dynamics Analysis Package, Fluid Dynamics International Inc. (2010).

[10] Błazik-Borowa E., Flaga A.: Interference galloping of two Identical Circular Cylinders at Unsteady Air Onflow, In: 2EACWE, Genua 22-26 June 1997, 1997, pp. 1815-1822.

[11] Mittal S., Kumar V.: Flow-induced vibrations of light circular cylinder at Reynolds number $10^{3}$ to $10^{5}$, Journal of Sound and Vibration 245(5), 2001, pp. 923-946.

[12] Lewandowski R.: An application of Hartlen-Curie model and the finite element method to transient and steady state analysis of vortex-induced vibration of beam, In: Proceedings of the Second East European Conference on Wind Engineering, Prague 7-11 September, Vol. 1, 1998, pp. 121-126.

[13] Beretta S. L.: Studio di uno schema numerico per la simulazione di un cilindro oscillante in una corrente fluida a bassi numeri di Reynolds. PhD thesis, Politecnico di Milano, 2007.

[14] Zienkiewicz O. C., Taylor R. L.: The finite element method (5th edition), Vol. I,II,III, Butterworth-Heinemann, 2000.

[15] NX/Nastran, Basic Dynamic Analysis User's Guide. UGS Corp, 2007.

[16] Hodges, D. H., Pirce D. A.: Introduction to structural dynamics and aeroelasticity, Cambridge Aerospace Series, Cambridge University Press, 2001.

[17] Introl, Corporate catalogue, Control-Measurement Apparatus. 
Numeryczna analiza drgań czujników uśredniających ciśnienie dynamiczne

\section{Streszczenie}

W artykule zaprezentowano proces oddziaływania wymuszonych przepływem płynu wirów na drgania przepływomierzy spiętrzających. Zaproponowany model matematyczny przepływu porównano z wynikami symulacji numerycznych w formie analizy modalnej. Częstotliwość generowania i odrywania się wirów policzono dla różnych prędkości przepływu dla czujnika jedno- i dwuprofilowego. Przeprowadzone analizy modalne umożliwiły wyznaczenie ich częstotliwości, a w rezultacie pomogły w wyznaczeniu prędkości przepływu, dla których analizowany czujnik podatny jest na zjawisko rezonansu. 